\title{
James Gregory: A Study in the Early History of Interpolation
}

By Professor H. W. Turnbull, F.R.S. (University of St Andrews).

(Received 15th November, 1932. Read 20th January, 1933.)

\section{INTRODUCTION.}

§ 1. IN a highly interesting critical account of the mathematical work of James Gregory (1638-1675), written for the Proceedings of the Edinburgh Mathematical Society, (1) 4I (1923), 2-25, by the late Professor G. A. Gibson, there occurs at p. 8 something of a mathematical puzzle. On that page a pair of formulæ are quoted, which certainly are striking examples of the analytical power of Gregory, and which run as follows :-

If $e=1-\cos c=2 \sin ^{2} \frac{1}{2} c$, then

$$
\begin{aligned}
\cos a=1-\frac{a^{2}}{2 c^{2}}(2 e)+\frac{a^{2}\left(a^{2}-c^{2}\right)}{4 ! c^{4}}(2 e)^{2}-\frac{a^{2}\left(a^{2}-c^{2}\right)\left(a^{2}-4 c^{2}\right)}{6 ! c^{6}}(2 e)^{3} & \\
& +\frac{a^{2}\left(a^{2}-c^{2}\right)\left(a^{2}-4 c^{2}\right)\left(a^{2}-9 c^{2}\right)}{8 ! c^{8}}(2 e)^{4}-\ldots \ldots,
\end{aligned}
$$

and, again, if $e / d=2(1-\cos \theta)=2 \sin ^{2} \frac{1}{2} \theta$, then

$$
\begin{aligned}
\sin \frac{a \theta}{c}=\sin \theta \cdot\left\{\frac{a}{c}-\frac{a\left(a^{2}-c^{2}\right)}{3 ! c^{3}} \frac{e}{d}+\frac{a\left(a^{2}-c^{2}\right)\left(a^{2}-4 c^{2}\right)}{5 ! c^{5}} \frac{e^{2}}{d^{2}}\right. \\
\left.-\frac{a\left(a^{2}-c^{2}\right)\left(a^{2}-4 c^{2}\right)\left(a^{2}-9 c^{2}\right)}{7 ! c^{7}} \frac{e^{3}}{d^{3}}+\ldots\right\} .
\end{aligned}
$$

Gibson adds that "there is another formula (Rigaud, p. 207), but it is of a very complicated character and I do not reproduce it." It will be convenient to refer to the above pair as formulæ $\mathrm{A}^{\prime}$ and $\mathrm{B}^{\prime}$, and to the more complicated but analogous series as formula $\mathrm{C}$, and to the original series, from which the above were transcribed, as formulæ $A$ and $B$. $\mathrm{I}$ am indebted to $\mathrm{Mr} \mathrm{A}$. Inglis for drawing my attention to the problem.

The puzzle consists in trying to discover how Gregory hit upon these three results: for, as Gibson remarks, Gregory gives no hint of the method by which he was led to them. The problem becomes still more interesting when we read of series $A$ ' that Gregory "has not expressed the coefficients in what would now be considered their simplest 
form." But if this is so, how indeed was the original of series $A^{\prime}$ constructed? And, further, if the series $C$ is still more complicated, how is its origin to be explained?

It is the purpose of this communication to propose a tentative solution, and to give reasons, both mathematical and historical, upon which the inference is based : for I cannot resist drawing the conclusion the Gregory had already made an independent discovery of a certain result in the theory of interpolation, nowadays called the Newton-Gauss Interpolation Formula, and that he was putting his knowledge into practice. Indeed it is a fact, as I shall show, that formula $\mathrm{C}$ is a straightforward illustration of the Newton-Gauss theorem, and that $A$ and $B$, the original forms of $\mathrm{A}^{\prime}$ and $\mathrm{B}^{\prime}$ are particular cases of the more general formula $\mathrm{C}$.

The historical interest of this work of Gregory is considerable; for the year 1670 , to which it may be referred, is a critical date in the prelude to the unfortunate story of the Newton-Leibniz controversy. Forty-two years later the endeavour of loyal admirers to establish Newton as the true inventor of Analysis (Infinite series and the Differential and Integral Calculus) culminated in a remarkable manifesto-the Commercium Epistolicum (London, 1712)-which was edited by a committee of the Royal Society. This contained many extracts of letters and other mathematical communications, dating from 1669 onwards, which passed between Barrow, Gregory, LeIBNiz, Newton, and several other eminent mathematicians, including JoHN CoLLINs (1625-1683), then Secretary of the Royal Society, who has been aptly called the Mersenne of English mathematics, playing as he did the part of an intermediary between geometers of England, Scotland, and the Continent. It was his habit to communicate many a fresh discovery as it came to his notice-although it has been sometimes too hastily assumed that he communicated everything that came his way, which was not the case. On the basis of the many notable papers, which Collins assiduously collected, this Commercium Epistolicum was prepared and published. But feeling ran high; and it was evidently not an unimpassioned publication such as might be expected to give the gist of the mathematical correspondence in its true perspective. By weighting everything in favour of Newton (who surely was the last man 
to need such adventitious help), it disparaged the work of others, and in particular that of Gregory: so much so that, until Gibson undertook the inquiry, historians appear always to have followed the implications of this somewhat partial account without ever correcting it by studying an alternative and instructive two-volume work, the Correspondence of Scientific Men of the Seventeenth Century, by Rigaud $(2,174-281)$. It is from these volumes, which give in fair completeness the mathematical material actually received by Collins, that Professor Gibson has constructed the account of Gregory from which the cited formulæ $\mathrm{A}, \mathrm{B}$, and $\mathrm{C}$ are taken. The conclusion to be gathered from a careful reading of the argument is somewhat to this effect: that in the five years 1665-1670, when Gregory and Newton were still young men (Gregory being two years older than Newton) they were working along parallel paths in mathematical analysis, and were discovering many theorems in complete independence of each other-though not of course independent of their common mathematical heritage. Among such theorems are the Binomial Theorem for fractional indices, and the analogous interpolation formula

$$
f(x)=f(b)+\frac{x-b}{h} \Delta f(b)+\frac{x-b}{h} \frac{x-b-h}{2 h} \Delta^{2} f(b)+\ldots
$$

(now known as the Gregory-Newton Formula), where $\Delta, \Delta^{2}, \ldots$, are the signs for successive differences at equal intervals $h$ of the argument $b$.

I am convinced that the verdict of Gibson is correct, and that it was indeed a misfortune to Scottish mathematics when James Gregory died in his thirty-seventh year, in the prime of his career. Gibson based his conclusions upon four short books-(i) the Optica Promota (London, 1660), (ii) Vera Circuli et Hyperbolce Quadratura (Padua, 1667), (iii) Geometrice Pars Universalis (Padua, 1668), (iv) Exercitationes Geometrica (London, 1668)-written by Gregory, together with the evidence of the Commercium and the Rigaud Correspondence. The favourable opinion which Gibson has formed is entirely in keeping with the further evidence which may be found in the extant letters of Collins to Gregory, and in the interesting notes which Gregory scribbled on the backs of these letters. They tell a very different story of his originality and resource from that, at any rate, of the later editions of the Commercium. 
Let us now pass on to the mathematics of the formulæ, and consider a way in which they might well have been evolved.

$$
\text { The Formuloe } A, B, C \text {. }
$$

$\S 2$. It is essential for my argument to quote the results in their traditional form - that is, in the form in which they occur in the Rigaud Correspondence (2, 204-207). On this authority we learn that the following formulæ, among many others, were transcribed by Collins from one or more letters dated 23rd November 1670 (and thereabouts) which he had received from Gregory. I shall follow Gibson in translating the Latin freely into English, but shall retain the original symbols $a, b, c, e, \ldots$ that occur, together with the word sinus (which connotes the product of the radius and the sine of an arc).

\section{Formula $A$.}

Gregory deals with two ares of a circle of radius $b$. Let the first arc be less than a quadrant by an amount $c$, and the second more than a quadrant by an amount $a$. Also let $b$ exceed the sinus of the first arc by $e$.

[If $a, 2 \beta$ are the corresponding angles at the centre subtended by these $\operatorname{arcs} a, c$, then evidently

$$
\left.b a=a, \quad 2 b \beta=c, \quad e=b(\mathbf{1}-\cos 2 \beta)=2 b \sin ^{2} \beta .\right]
$$

Let there be two sequences of continued proportions

and

$$
b, \quad 2 e, \quad 4 e^{2} / b, \quad 8 e^{3} / b^{2}, \quad 16 e^{4} / b^{3}, \ldots .
$$

$$
-e, \quad-2 e^{2} / b, \quad-4 e^{3} / b^{2}, \quad-8 e^{4} / b^{3}, \quad-16 e^{5} / b^{4}, \ldots,
$$

and let there be two further sequences, of which the first is

$$
\frac{a^{2}-c^{2}}{1.2 . c^{2}}, \quad \frac{a^{2}-a c-1 \cdot 2 c^{2}}{3.4 . c^{2}}, \quad \frac{a^{2}-a c-2.3 c^{2}}{5.6 . c^{2}}, \quad \frac{a^{2}-a c-3.4 c^{2}}{7.8 . c^{2}}, \ldots
$$

Let $\frac{o}{c}, \frac{p}{c}, \frac{q}{c}, \frac{z}{c}, \frac{o^{\prime}}{c}, \ldots$, respectively, denote the first term, the product of the first two, the product of the first three terms, etc. Next, let the second sequence be

$$
\frac{a}{c}, \quad \frac{a^{2}-c^{2}}{2.3 c^{2}}, \quad \frac{a^{2}-4 c^{2}}{4.5 c^{2}}, \quad \frac{a^{2}-9 c^{2}}{6.7 c^{2}}, \quad \text { etc. }
$$


and let the product of the first two terms be $\frac{r}{c}$, that of the first three be $\frac{s}{c}$, that of the first four $\frac{t}{c}$, etc. Then that arc which exceeds the quadrant by an amount $a$ will have for its sinus

$$
b-\frac{2 o e+a e}{c}+\frac{4 p e^{2}+2 r e^{2}}{b c}-\frac{8 q e^{3}+4 s e^{3}}{b^{2} c}+\frac{16 z e^{4}+8 t e^{4}}{b^{3} c}-\frac{32 o^{\prime} e^{5}+16 v e^{5}}{b^{4} c}+\text { etc. }
$$

This very prolix enunciation has brought us to a series which I shall call A. It is the strangely complicated form which Gibson has duly transcribed-after taking the obviously legitimate step of writing $b=1$-into the more familiar shape $A^{\prime}$ as already cited. If the above instructions are carried out the result is

$$
\begin{aligned}
b \sin \left(\frac{\pi}{2}+\frac{a}{b}\right)=b- & \left(\frac{2\left(a^{2}-c^{2}\right)}{2 ! c}+a\right) \frac{e}{c} \\
& +\left(\frac{4(a+c) a(a-c)(a-2 c)}{4 ! c^{3}}+\frac{2 a\left(a^{2}-c^{2}\right)}{3 ! c^{2}}\right) \frac{e^{2}}{b c}-\ldots
\end{aligned}
$$

\section{Formula $B$.}

$\S 3$. This deals with an arc whose sinus is $d$, while $2 d-e$ is that of the double arc. [If, as Gibson writes it, $\sin \theta=d$, and $b=1$, then $e / d=$ $2(1-\cos \theta)$; and Gregory now takes a third arc subtending an angle $a \theta / c$ at the centre of the circle.] The actual formula is

$$
\frac{a d}{c}-\frac{b e}{c}+\frac{k e^{2}}{c d}-\frac{l e^{3}}{c d^{2}}+\ldots
$$

which gives the value of $\sin (a \theta / c)$, where $b, k, l \ldots$, have precisely the significance of the $r, s, t, \ldots$, in the earlier case.

[Here I suspect that Gregory wrote $h, k, l, \ldots$ and that the $b$ is transcribed in error. Curiously enough Collins actually warned him in his letter of 24th December 1670 of the danger lurking in the written $b$, which might easily be confused with the figure 6 . It is also perhaps of interest to state that in the original document Gregory probably wrote his indices alongside, and not above, the letters which they modify:

$$
\left.\frac{k e 2}{c d}-\frac{l e 3}{c d 2}\right]
$$


Without any further reduction, beyond making the appropriate substitutions from the generating sequence (4), this series B is identical with series $B^{\prime}$ as originally quoted, term for term, and factor for factor. Gregory illustrates his series by taking $a=1, c=225$. Had he taken $a$ to be a multiple of $c$ the resulting series would have been finite. Clearly Gregory was interested in the infinite case.

It will be noticed that, whereas series $A$ was specified by two progressions (1) and (2), and two generating sequences (3) and (4), the series $B$ requires only one progression and one sequence.

\section{Formula $C$.}

$\S 4$. Let there be three arcs [of a circle of radius $\rho$ ] in arithmetical progression with a common difference $c$; and let the sinus be $(b-e-d)$, $b,(b+d)$ respectively, and an arbitrary arc be $a$.

[In other words, let

$$
\begin{aligned}
& \rho \sin (\alpha-2 \beta)=b-e-d, \\
& \rho \sin a=b, \\
& \rho \sin (\alpha+2 \beta)=b+d,
\end{aligned}
$$

in terms of corresponding angles in arithmetical progression at the centre.]

From a slightly modified pair of progressions $b, e, e^{2} / b, e^{3} / b^{2}, \ldots$ and $d, e d / b, e^{2} d / b^{2}, \ldots$, together with the same sequences (3) and (4) Gregory forms the following relation:-

$$
\text { Sinus arcus } a=\left\{\begin{array}{l}
b-\frac{e o}{c}+\frac{e^{2} p}{b c}-\frac{e^{3} q}{b^{2} c}+\frac{e^{4} z}{b^{3} c}-\ldots \\
+\frac{d a}{c}-\frac{e d r}{b c}+\frac{e^{2} d s}{b^{2} c}-\frac{e^{3} d t}{b^{3} c}+\ldots
\end{array}\right.
$$

the right-hand member of which I shall call formula $\mathrm{C}$. He adds that there are boundless other different ways of measuring circular arcs, many of which he illustrates. They mostly depend upon other principles such as the Vieta method of repeated bisection, or the expansion of a sine or inverse sine as a power series: and therefore they do not concern us here.

If these three series A, B, C are compared, it is at once obvious that the peculiar structure of A, already noticed by Gibson, is equally 
resident in the last series C. In expressing A Gregory has merely collected pairs of terms according to powers of $e$-while in $\mathrm{C}$ he has left them uncollected. In other words, the series $\mathrm{C}$ is less sophisticated and more original than the series $\mathrm{A}$, and therefore invites inspection. But unfortunately at this point quite an unexpected difficulty confronts the investigator, for the simple reason that, as it stands, this formula is wrong: left and right members of the equation fail to agree! Presumably, in view of the careful prescription which Gregory has formulated for the structure of the actual series, the error lies in the left rather than the right of the equation. But this raises the interesting question: How should the left expression, sinus arcus $a$, be modified?

In modern notation this expression is $\rho \sin (a / \rho)$ : which may be satisfactorily corrected by writing

$$
\rho \sin \left(a+\frac{a}{\rho}\right) .
$$

Evidently the angle $\alpha$, subtended by the mean of the three given arcs has accidentally dropped out of the formula: and most probably Gregory himself made the slip. It is unlikely that either Collins or Rigaud has erred, since the transcription of this restored expression $\mathrm{C}^{\prime \prime}$ into the original enunciation would be very involved, the actual angle (or arc) a not having been explicitly mentioned.

The error is one that might easily be made-like that of referring the coordinate of a point to two different origins in the same formula. There are three fixed arcs, let us say $A P, A Q, A R$, in arithmetical progression (so that $Q$ is midway along the arc $P R$ ), together with a variable arc $A X$. The confusion may have arisen by measuring the variable arc, on the left of the identity from $A$, and on the right from $Q$-and calling it $a$ in each case.

\section{The Newton-Gauss Formula.}

$\S 5$. To explain how I think that Gregory discovered his series it is first necessary to state the Newton-Gauss formula. In terms of the successive differences $\Delta, \Delta^{2}, \ldots$, of a function $f(x)$ whose value is known at each of the particular arguments

$$
\ldots, a-2 \beta, \quad a, \quad a+2 \beta, \quad a+4 \beta, \ldots,
$$


this formula may be written

$$
\begin{aligned}
f(a+2 x \beta)=f(\alpha)+ & x \Delta f(a)+\left(\begin{array}{c}
x \\
2
\end{array}\right) \Delta^{2} f(\alpha-2 \beta) \\
& +\left(\begin{array}{c}
x+1 \\
3
\end{array}\right) \Delta^{3} f(\alpha-2 \beta)+\left(\begin{array}{c}
x+1 \\
4
\end{array}\right) \Delta^{4} f(\alpha-4 \beta) \\
& +\left(\begin{array}{c}
x+2 \\
5
\end{array}\right) \Delta^{5} f(\alpha-4 \beta)+\left(\begin{array}{c}
x+2 \\
6
\end{array}\right) \Delta^{6} f(\alpha-6 \beta)+\ldots
\end{aligned}
$$

where

$$
\left(\begin{array}{l}
x \\
r
\end{array}\right) \equiv \frac{x(x-1) \ldots(x-r+1)}{1.2 \ldots r}
$$

Whereas $r$ is a positive integer, $x$ may be fractional, or even irrational: $\left(\begin{array}{l}x \\ r\end{array}\right)$ is in fact a general binomial coefficient.

The characteristic feature of this formula is the rise in value of the upper index of the binomial coefficient-in contrast to the behaviour of the binomial series and. of the Gregory-Newton formula, both of which have a fixed upper index. But this rise of index is precisely the feature of each formula $A, B, C$, as may at once be seen if we replace the homogeneous expressions (with which Gregory habitually worked) by writing

$$
x=\frac{a}{c}
$$

In this notation his products of sequences (3), (4) become

and

$$
\left.\frac{o}{c}, \quad \frac{p}{c}, \quad \frac{q}{c}, \ldots=\left(\begin{array}{c}
x+1 \\
2
\end{array}\right), \quad\left(\begin{array}{c}
x+2 \\
4
\end{array}\right), \quad\left(\begin{array}{c}
x+3 \\
6
\end{array}\right), \ldots\right)
$$

$$
\left.\frac{r}{c}, \quad \frac{s}{c}, \quad \frac{t}{c}, \ldots=\left(\begin{array}{c}
x+1 \\
3
\end{array}\right), \quad\left(\begin{array}{c}
x+2 \\
5
\end{array}\right), \quad\left(\begin{array}{c}
x+3 \\
7
\end{array}\right), \ldots\right\}
$$

But these are typical of the coefficients required in the odd and in the even terms of the Newton-Gauss formula. Furthermore, the usual way of applying this formula to a function $f(a+x \omega)$ is first to form a table of differences $\Delta f, \Delta^{2} f, \ldots$ arranged in columns, then to select two consecutive rows of entries in this table, and then to multiply them, term by term, by the binomial coefficients of the array (2). On the supposition that the said rows happen to be terms of two geometrical progressions this is, as we have seen, precisely the procedure adopted 
by Gregory for constructing both his series A and C. But this is the property of the circular functions $\sin \alpha$ and $\cos \alpha$-as already had been remarked by BRIGGS on p. 30 of his first edition of the Arithmetica Logarithmica, in the year 1624 . It is natural to suppose that Gregory also knew this fact, although he may not have known that it was familiar to Briggs. At any rate the following mathematical procedure, which was well within the competence of Gregory, explains the generation of his series-even to the very topological arrangement of their terms.

The Difference Table for $\sin \alpha$.

$\S 6$. If the following notation is employed

$$
u=2 \sin \beta, \quad c_{n}=\cos (\alpha+n \beta), \quad s_{n}=\sin (\alpha+n \beta),
$$

then the fundamental formule for differences of sines and of cosines can be written.

$$
c_{n}-c_{n-2}=-u s_{n-1}, \quad s_{n}-s_{n-2}=u c_{n-1},
$$

as may at once be verified. If $n$ is allowed to take all positive and negative integral values it is easy now to construct a difference table for the function $\sin (\alpha+2 x \beta)$, namely:

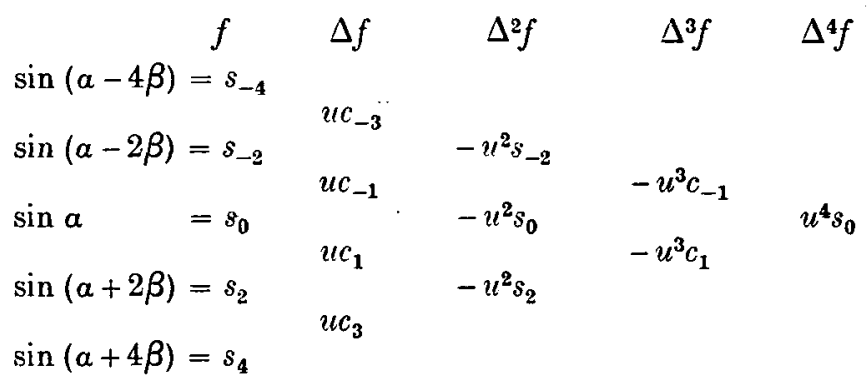

Any entry $\Delta^{i} f$ is formed by subtracting an $(i-1)^{\text {th }}$ difference from that immediately below it, and then placing the result, the $i^{\text {th }}$ difference, midway between but on their right. Such a table evidently generates sequences such as $s_{0},-u^{2} s_{0}, u^{4} s_{0}$ which are in geometrical progression ; indeed, every row of entries indefinitely continued possesses this property-as Briggs explicitly stated.

Next from two consecutive rows we find that $\Delta \sin a=u c_{1}$, $\Delta^{3} \sin (\alpha-2 \beta)=-u^{3} c_{1}, \Delta^{5} \sin (\alpha-4 \beta)=u^{5} c_{1}, \ldots, \Delta^{2} \sin (\alpha-2 \beta)=-u^{2} s_{0}$, $\Delta^{4} \sin (\alpha-4 \beta)=u^{4} s_{0}, \ldots$ 
If these are substituted in the Gauss formula (with $\sin a$ for $f(a)$ and $a / c$ for $x$ ) we obtain at once

$$
\begin{aligned}
\sin \left(a+2 \frac{a}{c} \beta\right)=\sin a(1 & \left.-\frac{a(a-c)}{2 ! c^{2}}(2 \sin \beta)^{2}+\frac{(a+c) a(a-c)(a-2 c)}{4 ! c^{4}}(2 \sin \beta)^{4}-\ldots\right) \\
& +\cos (a+\beta)\left(\frac{a}{c}(2 \sin \beta)-\frac{(a+c) a(a-c)}{3 ! c^{3}}(2 \sin \beta)^{3}+\ldots\right) .
\end{aligned}
$$

It will be noted that each of these two series ascends in powers of $(2 \sin \beta)^{2}$. After using the identity

$$
2 \cos (\alpha+\beta) \sin \beta=\sin (\alpha+2 \beta)-\sin \alpha
$$

let us write the result

$$
\sin \left(\alpha+2 \frac{a}{c} \beta\right)=(\sin a) U+(\sin (\alpha+2 \beta)-\sin \alpha) V,
$$

where $U$ and $V$ are power series in $(2 \sin \beta)^{2}$, starting with initial terms 1 and $\frac{a}{c}$ respectively. But the series $\mathrm{C}$ of Gregory is also such a pair of power series in $e / b$. If therefore we take

$$
u^{2}=(2 \sin \beta)^{2}=e / b,
$$

then we have at once (in the previous notation)

$$
\begin{aligned}
& U=1-\frac{o}{c} \frac{e}{b}+\frac{p}{c}\left(\frac{e}{b}\right)^{2}-\frac{q}{c}\left(\frac{e}{b}\right)^{3}+\ldots \\
& V=\frac{a}{c}-\frac{r}{c} \frac{e}{b}+\frac{s}{c}\left(\frac{e}{b}\right)^{2}-\frac{t}{c}\left(\frac{e}{b}\right)^{3}+\ldots \\
& C=b U+d V
\end{aligned}
$$

Clearly we can now evaluate the Gregory series $C$ by taking $b=\rho \sin a$, $d=\rho(\sin (\alpha+2 \beta)-\sin \alpha)$ in the above relation (1), in agreement with the previous relation $\S 4(1)$.

This gives

$$
\rho \sin \left(a+\frac{2 \alpha \beta}{c}\right)=b U+d V=C .
$$

But by definition $c=2 \rho \beta, \rho$ being the radius and $c$ the arc subtended by the angle $2 \beta$. Hence, finally,

$$
C^{\prime \prime} \equiv \rho \sin \left(a+\frac{a}{\rho}\right)=b U+d V=C \text {. }
$$


This proves that the Gregory series $\mathrm{C}$ is in fact the expansion of a sine by the Newton-Gauss formula, the terms being written in their natural order in relation to the difference table of sines.

It is now interesting to see how naturally the two other series follow, by taking for $a$ the special values $\frac{\pi}{2}$ and zero.

(i) Series $A^{\prime}$. - If $a=\frac{\pi}{2}$, then on substitution in (1) we have

$$
\sin \left(\frac{\pi}{2}+\frac{a}{\rho}\right)=\sin \frac{\pi}{2} \cdot U+\left(\sin \left(\frac{\pi}{2}+2 \beta\right)-\sin \frac{\pi}{2}\right) V .
$$

In this case Gregory writes $b$ for the radius $\rho$, and introduces $e$ by the formula

$$
e=b(1-\cos 2 \beta) \text {. }
$$

Hence $(2 \sin \beta)^{2}=2 c / b$, which accounts for the duplication of numerical coefficients in his sequences $b, 2 e, 4 e^{2} / b, \ldots$, etc. On making these substitutions in (4) and multiplying through by $b$, the Gregorian formula at once falls out: on the left $b \sin \left(\frac{\pi}{2}+\frac{a}{\rho}\right)$ is precisely what he calls "the sinus of that arc which exceeds the quadrant by an amount $a$ " (Arcus ille, quem superat quadrans excessu $a$, habebit sinus $=\ldots$.) : while on the right, when terms are grouped in powers of $e$, there is

$$
b-\frac{2 o e+a e}{c}+\frac{4 p e^{2}+2 r e}{c b}-\ldots .
$$

which is the series A.

(ii) Series $B^{\prime}$.-If $a=0$, then (2) becomes $\rho \sin \frac{2 a \beta}{c}=d V$, since the coefficient of $U$ vanishes. Here Gregory defines $d$ and $e$ by the equivalent of the following conditions:-

$$
d=\rho \sin 2 \beta, \quad 2 d-e=\rho \sin 4 \beta:
$$

that is, $e / d=(2 \sin \beta)^{2}$, as in the original $C$. On expressing the series $V$ in terms of $a, d, c, e, r, \ldots$, the formula yields

$$
\begin{aligned}
\rho \sin \frac{2 a \beta}{c} & =\frac{a d}{c}-\frac{r e}{c}+\frac{s e^{2}}{d c}-\frac{t e^{3}}{c d^{2}}+\ldots \\
& =\frac{a d}{c}-\frac{b e}{c}+\frac{k e^{2}}{c d}-\frac{l e^{3}}{c d^{2}}+\ldots .
\end{aligned}
$$


in the literal notation of Rigaud as already quoted-that is, the Gregorian series B.

Summing up, we therefore see that the three series, C, A, B, are in fact the Newton-Gauss expansions of the function

$$
\rho \sin \left(a+\frac{a}{\rho}\right)
$$

whose argument is $a$, for (i) the general constant angle $a$, (ii) the value $\alpha=\pi / 2$, and (iii) the value $\alpha=0$ respectively.

\section{The Gregory Newton Formula and the Binomial Theorem.}

$\S 7$. The conclusion which has just been reached on mathematical grounds is indirectly considerably strengthened when we reflect that an example of the other fundamental formula of interpolation-the Gregory-Newton formula-is quoted on p. 211 of Rigaud from the very same passage of Gregory's work in which the series A, B, C occur. So too is an example of the binomial theorem for a fractional index. This occurs on p. 212. But if the view already adopted is correctwith.its implication that Gregory was familiar with the behaviour of a difference table, - then it is natural to suppose that he would test not only the trigonometrical sine but also the simpler algebraic function $a^{x}$ by such a table. Just as $\sin (a+2 x \beta)$ leads to geometrical progressions in the horizontal rows of the table, so too $a^{\alpha+x \beta}$ leads to diagonal geometrical progressions. But while the horizontal entries are needed for the Gauss formula, the diagonal entries are needed for the GregoryNewton formula. It is tempting, therefore, to suppose that Gregory discovered the binomial theorem by applying his interpolation formula to the table in which the column of entries $f(a)$ were a set of numbers in geometrical progression. In illustration of this supposition, I shall quote the passage (Rigaud, p. 212; Gibson, p. 5) at length.

"Given $b, \log b=e, b+d, \log (b+d)=e+c$, it is required to find the number whose logarithm is $e+a$.

Take the series of continued proportionals

and another series

$$
b, \quad d,=d^{2} / b, \quad d^{3} / b^{2}, \ldots .
$$

$$
\frac{a}{c}, \quad \frac{a-c}{2 c}, \quad \frac{a-2 c}{3 c}, \ldots \frac{a-3 c}{4 c}, \ldots
$$


let $f / c$ be the product of the first two terms of the second series, $g / c$ that of the first three, $h / c$ that of the first four, $i / c$ that of the first five, and so on. The number will be $e+a$, where

$$
e+a=b+\frac{a d}{c}+\frac{f d^{2}}{c b}+\frac{g d^{3}}{c b^{2}}+\frac{h d^{4}}{c b^{3}}+\frac{i d^{5}}{c b^{4}}+\frac{k d^{6}}{c b^{5}}+\ldots
$$

Now if we put their values for $f / c, g / c$, etc., the series is

$$
b+\frac{a}{c} d+\frac{a(a-c)}{c .2 c} \frac{d^{2}}{b}+\frac{a(a-c)(a-2 c)}{c .2 c \cdot 3 c} \frac{d^{3}}{b^{2}}+\ldots
$$

and this is simply $b\left(1+\frac{d}{b}\right)^{\frac{a}{c}}$. That this is the solution desired is ohvious since

$$
\log \left\{b\left(1+\frac{d}{b}\right)^{\frac{a}{c}}\right\}=\log b+\frac{a}{c}\{\log (b+d)-\log b\}=e+a .
$$

Gregory adds the remark, "Hence with a little work but without difficulty any pure equation whatever may be solved."

I had already come to the conclusion that Gregory had utilized the Gauss formula before I reached this further example of his methods, which it will be noted occurs on a later page in Rigaud. But the example D shows exactly the same general features as before-even to the notation $a / c$. This affords a strong presumption that all the said formulæ were discovered by the same methods: and I have little doubt that the difference table was the clue. Just as the earlier examples fell out term for term by assuming a working knowledge of the Gauss formula, so here the above prescriptions-(1) and (2) and what follows them-are a direct statement of the Gregory-Newton formula in its application to a function $f$ whose differences $\Delta f(a), \Delta^{2} f(a), \ldots$, for unchanged argument, are in geometrical progression.

It may be objected that the exponential function $a^{x}$, which must be postulated for this particular difference table, is somewhat farfetched. Surely Gregory would be more likely to try $x^{a}$ first. To this the answer is simple. He did-on p. 211 of Rigaud. Gregory gave the cube of 23 by applying his formula to the difference table for $10^{3}$, $15^{3}, 20^{3}, 25^{3}, 30^{3}$, where

$$
\Delta f(10)=2375, \quad \Delta^{2} f(10)=2250, \quad \Delta^{3} f(10)=750 ;
$$


whence

$$
f(23)=1000+6175+4680+312=12167 .
$$

This is evidently an application of the Gregory-Newton formula to a terminating difference table-terminating because all differences after those of a certain order (here the third) are zero.

All the series with which we are dealing can be put into the form

$$
c_{0} \Delta_{0}+c_{1} \Delta_{1}+c_{2} \Delta_{2}+c_{3} \Delta_{3}+\ldots \ldots \text {, }
$$

where the $c_{i}$ are suitable coefficients, polynomials in $a / c$, and the $\Delta_{i}$ are certain $i^{\text {th }}$ differences. Such a series may terminate for either of two reasons: (i) if all the $\Delta_{i}$, or (ii) if all the $c_{i}$ vanish after a finite term. Thus the series for $23^{3}$, an essentially finite series, is an example of the former, while the series A, B, C, D would be examples of the latter, although they do not invariably remain finite, but only when $a / c$ is an integer. It would not be hard to give examples from the work of Briggs, Vieta, and Pascal of series very like these in their finite case. What distinguishes the work of Gregory, and is in fact the chief interest of his series, is that he explicitly uses them in their infinite form.

The reader may wonder if Gregory tested the other trigonometrical functions in a similar way to the sine. The cosine provides nothing new ; but it is highly interesting to find on the back of Collins's letter, dated 15th December 1670, the expansions for the tangent and the secant analogous to formula $\mathrm{C}$, but naturally lacking the same elegance. These, I think, were never published.

\section{Historical Survey.}

\$ 8. In studying the mathematics of the seventeenth century it is always difficult to ascertain how far the results in those days were independent discoveries. There is, for example, a strong likeness between this work of Gregory and that of Briggs, of Vieta, of Alexander Anderson (who edited Vieta's trigonometry), and of Isaac Newton, each of whom was interested in interpolation or in trigonometry. BRIGGS, the friend and successor of Napier, the highly gifted computator who brought Napier's work to fruition, has handed down in his introductory chapters to the Arithmetica Logarithmica (1624) and the Trigonometria Britannica (1633) the precise rules by which he was guided in his 
calculations. How he went to work does not well appear: he only set forth the rules, but not the investigation of them. But they are remarkable, although they are couched in arithmetical language, rather than in algebraic symbols, a fact which has probably hindered them from becoming better known. He gives, for example, the GregoryNewton formula for the case when third and higher differences are negligible (Logarithmica, chap. xii, p. 24 (1624)), with a rider to effect the solution of the problem when second differences are no longer equal but nearly so. In the next chapter he gives a formula which can best be stated as follows:-

$$
\Delta^{n} u_{0}=c_{0} \delta^{5 n} u_{0}+c_{1} \delta^{5 n-2} u_{1}+c_{2} \delta^{5 n-4} u_{2}+\ldots+c_{2 n} \delta^{n} u_{2 n},
$$

where $\Delta u_{0}=u_{5}-u_{0}, \delta u_{0}=u_{1}-u_{0}$, and where the $c_{i}$ are certain numerical coefficients. He states this formula in arithmetical language-that is, Briggs gives a precise rule for writing down such a series for low values, for $n=1,2,3,4$ in fact, together with a table or abacus for the $c_{i}$, somewhat like that of the figurate numbers (the Pascal triangle). Remarkable as this formula is, it is unlikely to have influenced the work of Gregory, as it has no obvious connexion with the usual interpolation formulæ. As Legendre * has virtually pointed out, this formula of Briggs is equivalent to the expansion of $\left\{\left(x^{5}-1\right) /(x-1)\right\}^{n} x^{-2 n}$ in ascending powers of $z$, where $z=x+x^{-1}-2$, a problem in the multinomial theorem. Such a resolution into an algebraic expansion is effected by writing

$$
\Delta=e^{5 D}-1, \quad \delta=e^{D}-1, \quad x=e^{D},
$$

in the original formula, by a well-known symbolic device. Or, again, one may write $e^{i \theta}$ for $x$ and reduce the problem to an expansion of

$$
\left(\sin \frac{5 \theta}{2} \operatorname{cosec} \frac{\theta}{2}\right)^{n}
$$

in ascending powers of $(2 \sin \theta)^{2}$. This last form is remarkable in that it gives another example of a trigonometrical expansion in powers exactly similar to those employed by Gregory, but for a totally different reason.

Briggs furthermore states the corresponding results when $\Delta=e^{3 D}-1$

* Cf. Maurice, Journal of the Institute of Actuaries, 14 (1869), 1 et seq. 
-that is, in one case he deals with quinquesection of the coarser differences $\Delta$ into finer divisions $\delta$; and in the other trisection. It is after this, on p. 30 of chapter xiii, that he alludes to the characteristic finiteness of the difference successions (zeros following a final set of constant differences) for the case of a fixed positive integral power $x^{n}$ of the variable: and, as has already been mentioned, to the characteristic twin geometrical progressions running horizontally in the difference table for sines which are arranged by column in arithmetical progression of arc. He even says that it is sufficient that three consecutive entries be given, in order to obtain all the remote differences by rule of proportion. This train of thought is very near to that in the enunciation of the $\mathrm{C}$ series of Gregory ; and it may conceivably be the true origin of the whole matter. Nevertheless, in my judgment it is highly unlikely that Gregory ever saw this chapter of Briggs, since it appears only in the first edition (1624), being inexplicably eliminated from subsequent editions of the book by the Continental editor Vlacq; and there is no evidence that this first edition was ever in the library of the University at St Andrews, where Gregory occupied the Chair of Mathematics at the time of this particular discovery of his (1670). Also he was, as he confesses, poorly paid, and therefore unlikely to possess a copy of the first edition, which was rare, especially as the library contained the second. Of this omission on the part of Vlacq, Briggs very properly complains on p. 37 of his Trigonometria Britannica (1633), which for like reasons Gregory is unlikely to have seen at that date. On that page Briggs remarks that the omission was unwarranted, and seemed to be caused by an intentional desire to deprive him of the honour of the invention of interpolation by successive differences. Whether or not this is so, the Trigonometria is notable for containing $\begin{array}{rrrr}\ldots & 1 & 1 & 1 \\ \ldots . & 4 & 3 & 2 \\ \ldots . & 10 & 6 & 3 \\ \ldots & 20 & 10 & 4 \\ \ldots . . & \ldots & 10 & \ldots \\ \end{array}$ a very early instance-possibly the first-of the general rule for determining the binomial coefficient $\left(\begin{array}{l}n \\ r\end{array}\right)$. Briggs made a thorough survey of the table of figurate numbers, which later was studied in much the same way and probably in total independence by Pascal, a table

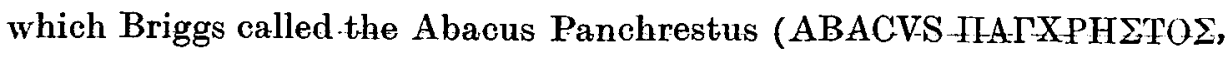
"the abacus good for all work"), a very shrewd prophecy. 
Incidentally he made a fertile contribution to the logic of mathematics by distinguishing between the genesis and the analysis of a mathematical expression, be it function or table of values. For example, in this abacus the genesis is specified by the addition law for forming successive entries downwards or to the left in the obvious way $(6=3+3,10=6+4)$. Evidently it is possible to extend the table by this method to any size-but the actual value of the general entry $\left(\begin{array}{l}n \\ r\end{array}\right)$ cannot yet be written down at a stroke. This is rather a property of the analysis of the table: namely,

$$
\left(\begin{array}{c}
n \\
r+1
\end{array}\right)=\left(\begin{array}{l}
n \\
r
\end{array}\right) \cdot \frac{n-r}{r+1} .
$$

To calculate this expression by running through the requisite terms in the table and adding them up would be to act genetically: to evaluate it from the single formula $n(n-1) \ldots(n-r) / 1.2 \ldots(r+1)$ would be to act analytically.

The same genesis applied to a modified gnomon of initial entries situated in the upper row and the right-hand column of a table leads to a new analysis. By replacing all the righthand column of integers by the odd integers and proceeding as before, Briggs obtains a new abacus which he at once employs for determining the length of a chord of a circular arc in terms

$\begin{array}{rrrrrr}\ldots & 1 & 1 & 1 & 1 & 1 \\ \ldots & 7 & 6 & 5 & 4 & 3 \\ \ldots & \ldots & 20 & 14 & 9 & 5 \\ \ldots \ldots & \ldots & \ldots \ldots & 30 & 16 & 7\end{array}$
of that of an odd submultiple arc; in other words, for determining the numerical coefficients $a_{i}$ in the expression

$$
2 \sin (2 r+1) \theta=a_{1}(2 \sin \theta)-a_{3}(2 \sin \theta)^{3}+a_{5}(2 \sin \theta)^{5}-\ldots
$$

summed to $r$ terms. Actually he writes the series in reverse order and in arithmetical notation. For example, he writes

$$
1(5)-5(3)+5 \text { (1) }
$$

meaning the series for $2 \sin 5 \theta$, which we should nowadays denote by

$$
2 \sin 5 \theta=1(2 \sin \theta)^{5}-5(2 \sin \theta)^{3}+5(2 \sin \theta) .
$$

His genetic rule, which is highly illuminating, comes to this: select the entries in the abacus that lie upon a diagonal descending to the right 
at a gradient of one in two-by knights' moves in fact-and ending on the final column: affix alternate signs, but always finishing with a positive sign at the final (right-hand) column. These are the required coefficients $(-)^{r} a_{2 r+1}, \ldots,-a_{3}, a_{1}$. In this way alternate and parallel diagonals are left out: but these in turn give the analogous coefficients for the square of $2 \sin r \theta$ for all positive integral values of $r$. Such diagonals finish at the entries in the second column from the right. For example, if $r=2$,

$$
(2 \sin 2 \theta)^{2}=-1(2 \sin \theta)^{4}+4(2 \sin \theta)^{2}=-1(4)+4(2):
$$

and the case following, when $r=3$, involves the coefficients $1,6,9$; and so on for higher values of $r$.

The series for $(2 \sin r \theta)^{2}$ is of course a series for $\cos 2 r \theta$ also; and by taking the series for $\sin (2 r+1) \theta$ and for $\cos 2 r \theta$ for ascending values of the integers $2 r, 2 r+1$, that is, in the order suggested by the abacus, a proof of either by induction is perfectly straightforward by use of the identity

$$
\sin (2 r+1) \theta-\sin (2 r-1) \theta=2 \sin \theta \cos 2 r \theta,
$$

and a similar one for the difference of cosines. Briggs has in fact given the genetic, rather than the analytic, mode of the inductive proof. But the whole treatment is very beautiful: and it is a pity that this presentation of the subject has, as far as I am aware, never figured in a text-book on trigonometry.

Had Briggs possessed a good algebraic notation he could easily have given the general analytical form for his coefficients-by adding together any adjacent pair in any column of the original abacus. Such a typical pair added together give

$$
\left(\begin{array}{c}
n+r-1 \\
r-1
\end{array}\right)+\left(\begin{array}{c}
n+r \\
r
\end{array}\right)=\frac{(n+2 r)(n+r-1) !}{n ! r !},
$$

where $n$ denotes the number of the column counted from the right, and $r$ the number of the row. For example, $n=3, r=3$; and $10+20$ in the original is equal to 30 in the modified abacus.

But we may go back a little further in the history and find the same work performed by VreTa (1540-1603), who was senior to Briggs 
by twenty-one years. The same arithmetical results for the values of $\sin (2 r+1) \theta$ and $\cos 2 r \theta$ appear, tabulated for successive values of $r=1,2,3, \ldots$ by means of a slightly modified arrangement, where any entry is determined by adding together that immediately above it and that two places above, but one place 2 to the left. Although this actual table only appears in 3 the 1646 Schooten edition of Vieta's Collected Works, at p. 295 in the chapter by Alexander Anderson, who here expounded Vieta, yet the whole theory was perfectly well known to Vieta in 1593, when he solved a celebrated problem of Adrian Romanus, at the behest of King

2
Henry IV of France, by rapidly calculating the above series for the chord of a circle when $2 r+1=45$. See, for instance, p. 319 of the same 1646 edition. On the whole I am inclined to think that, as in so many other cases, these were independent discoveries, priority resting with Vieta. There appear to be no cross-references in all these books, either of Briggs to Vieta or of Anderson to Briggs: and even the scholarly Wallis, who occupied the Savilian Chair at Oxford some twenty years after Briggs had vacated it, was unaware that Briggs had solved the problem of the figurate numbers which had baffled him, although he was familiar at any rate with part of Briggs's work.

The above description of the state of trigonometry in the first half of the seventeenth century throws light upon the problem of Gregory's series from which we started. It shows the general way in which such problems were likely to be attacked. Moreover, it is practically certain that Gregory was familiar with what Alexander Anderson, himself a brilliant geometer, had written about Vieta, seeing that Gregory was a near relative (Janet Anderson, who married into the Gregory family and was mother of James Gregory, was first cousin of Alexander Anderson). The latter had migrated from his Aberdonian home to become Professor of Mathematics in Paris, where it is likely that uncle and nephew would meet-although I cannot recall any record of such a meeting. This might well take place in the course of Gregory's travels between London and Padua, where he stayed for some few years and whence he returned through London to Aberdeen in 1668.

It is evident that there is a connexion between the Vieta-Briggs 
series and those of Gregory, more particularly if the latter are cast into their genetic arithmetical form-that is, if the ratio $a: c$ is made to take positive integral values $1,2,3, \ldots$ The abacus for the series $U$ and that for the series $\mathrm{V}$ can then be given as

$\begin{array}{rrrrrrrrrr}1 & & & & & 1 & & & & \\ 1 & 1 & & & & 2 & 1 & & & \\ \mathrm{U}: & 1 & 3 & 1 & & \mathrm{~V}: & 4 & 1 & & \\ 1 & 6 & 5 & 1 & 4 & 10 & 6 & 1 & \\ 1 & 10 & 15 & 7 & 1 & 5 & 20 & 21 & 8 & 1\end{array}$

and these are connected with the abacus of Vieta by forming the sum of consecutive rows both in $U$ and in $V$. Incidentally $U$ is a first difference table for the rows of $\mathrm{V}$, and vice versa. But this a posteriori recognition of a link between the theories cannot be taken as an argument that Gregory discovered his coefficients in $U$ and $V$ from a study of Vieta ; more particularly because there is no clear reason for explaining how the series for $\sin (2 r+1) \theta$ in powers of $\sin \theta$ should be modified into a series in powers of $\sin ^{2} \frac{\theta}{2}$ as in the series $A$ of Gregory. And even if it could be proved that Gregory had his arithmetical results from others, he at any rate was one of the first to throw them into analytical form, to quote the general series, and-what is still more characteristic of his contribution to mathematics-to apply them not to positive integral but to fractional values of the ratio $a: c$.

Another mathematician besides Briggs who knew the general value of $\left(\begin{array}{l}n \\ r\end{array}\right)$ was PASCAL, who introduced his arithmetical triangle in 1653, the results of which were first published in 1665. As Gregory was at that time on the Continent he would possibly become acquainted with the book and its contents. In an interesting Tract [12 (1812), 233], Hutton has remarked: "The contemplation of this table has probably been attended with the invention and extension of some of our most curious discoveries in mathematics." Certainly a list of pioneers who used this table would include Stifel, Cardan, Stevinus, Napier, Vieta, Briggs, Oughtred, Mercator and Pascal (I have added the name of Napier to Hutton's list). 


\section{Gregory and Newton.}

§9. It is appropriate to conclude this survey by giving a reason for supposing that this work of Gregory, which dates from his letter to Collins of 23rd November 1670, is independent of that of Newton, who is known to have been active in his analytical discoveries since the year 1665. The conclusion which Gibson reaches-namely, that previous to this letter Gregory had only received one single piece of mathematical information from Newton through Collins, is certainly borne out by a perusal of the actual letters of Collins to Gregory, which happily have been preserved in the University Library at St Andrews. For many years Collins and Gregory had maintained a regular and very friendly correspondence, at a time when Newton was a young and rather a shy scholar at Cambridge, of whom Collins had seen very little. In a letter of the 25th November 1669 he first mentions Newton to Gregory, when he tells that "Mr Barrow hath resigned his Lecturer's place to one Mr Newton of Cambridge whome he mentioneth in his Optick Praeface as a very ingenious person, one who (before Mercator's Logarithmotechnia was extant) invented the same method and applyed it generally to all Curves, and diverse wayes to the Circle, which possibly he may send up to be annexed to Mr Barrowes Lectures."

Again, in a letter on 24th March 1670 Collins writes to Gregory as follows: "Mr Newtone of Cambridge sent the following series for finding the Area of a Zone of a Circle to Mr Dary, to compare with the said Dary's approaches, putting $R$ the Radius and $B$ the parallel distance of a Chord from the Diameter the Area of the space or Zone between them is

$$
=2 R B-\frac{B^{3}}{3 R}-\frac{B^{5}}{20 R^{3}}-\frac{B^{7}}{56 R^{5}}-\frac{5 B^{9}}{576 R^{7}}, "
$$

And this appears to be the only clue to what Newton was achieving, which reached Gregory, until he had communicated in November his own results to Collins. In a reply, on the 23th December 1670, Collins wrote at great length, first thanking Gregory for his "excellent Papers," which he had "not yet thoroughly perused" (little wonder!); and then telling all the mathematical news, and incidentally mentioning that he had only twice met Newton: "somewhat late on a Saturday night at his Inne, I then proposed to him the adding of a Musicall Progression the which he promised to consider and send up .... and again I saw 
him the next day having invited him to Dinner." After describing in some detail typical series (such as the expansions for $\sin x, \sin ^{-1} x$ in powers of $x$ ) which Collins "had out of Newton's method," he goes on to say that he tried to draw Newton into making further disclosures: but "I received no more in answer than what I sent you, hence observing a warinesse in him to impart, or at least an unwillingness to be at the paines of so doing I desist, and doe not trouble him any more, and upon the whole I cannot as yet perceive your Methods to be the same with his, though both performe the same thing."

It would take one too far afield to deal with this most interesting correspondence at all thoroughly, particularly as it includes considerably more mathematics than the series $\mathrm{A}, \mathrm{B}$, and $\mathrm{C}$ which have prompted the foregoing survey. Other results, involving the above series for the Zone, and those for $\sin x$ and $\sin ^{-1} x$, to which Collins alludes, are to be found in the Rigaud extracts, and also in the account by Gibson. Others still, which perhaps never reached Collins, exist in the rough MS notes in Gregory's own handwriting preserved among these letters of Collins. Were they to be published they would make an interesting addition to our knowledge of that peculiarly fertile mathematical decade. Enough has been said to show that the estimate of Gibson was well founded, and that Gregory was indeed a geometer of the first rank.

The author desires to acknowledge with hearty thanks the generous help received from the University Court of St Andrews towards the cost of printing this communication.

\section{BIBLIOGRAPHY.}

GIBson, Proc. Edinburgh Math. Soc., I, 41 (1922), 2-25; and II, 1 (1927), $1-18$.

RIGAUD, Correspondence of Scientific Men of the Seventeenth Century, 2, 185-211.

Commercium Epistolicum D. Johannis Colline et Alioram de Analysi Promota (London, 1712).

BRIGGS, Arithmetica Logarithmica (London, 1624), 24-32.

BRIGGs, Trigonometria Britannica (1633), 12-52.

VIETA, Opera Mathematica: edited by Schooten (1646), 287-324.

Hotron, Tracts on Mathematical Subjects, 1, Tract 12, 228-244; Trạcts 19, $20,21$.

MaURIOE, Journal of the Institute of Actuaries, 14 (1869), 1, who quotes Legendre's Connaissance des Termes (1817).

WhItTAKer and RobINson, The Calculus of Obserrations (Glasgow, 1926), $1-52$. 